symptoms (RR 0.25 (0.10 to 0.60$)$ ) of C30 questionnaire. Emotional support was negatively associated with global health in women aged under 60 years (RR 0.65 (0.50 to 0.85$)$ ).

Conclusions In this population social environment is positively associated with QOL; this effect is greatest in elderly women.

\section{P2-80 EARLY COMPLICATIONS OF BREAST CANCER TREATMENT AND OUALITY OF LIFE IN BRAZILIAN WOMEN}

doi:10.1136/jech.2011.142976i.15

${ }^{1} \mathrm{D}$ B Ferreira, ${ }^{* 1} \mathrm{R}$ Koifman, ${ }^{2} \mathrm{~A}$ Bergmann. ${ }^{1}$ National School of Public Health, Oswaldo Cruz Foundation, Rio de Janeiro, Brazil; ${ }^{2}$ National Cancer Institute, Rio de Janeiro, Brazil

Introduction Post-operative complications are a major cause of morbidity in women with breast cancer. The aim of this study was to evaluate the association between quality of life (QOL) before treatment for breast cancer and early post-operative complications in the National Cancer Institute-Brazil.

Methods Participants were women with unilateral breast cancer, requiring oncological treatment in National Cancer Institute-Brazil between June 2009 and March 2010. QOL before treatment was assessed using EORTC QLQ-C30 and BR23 module. Information about early post-operative complications (axillary web syndrome, pain and subjective symptoms related to early oedema in the upper limbs) were obtained by physical examination 30 days after surgery, collected using a standardised form. Bivariate analyses of dichotomous variables and independent variables were carried out and ORs, with $95 \%$ CIs present.

Results 181 women were studied. Women who reported good health overall were $57 \%$ less likely to experience early subjective oedema ( $95 \%$ CI 0.20 to 0.96$)$. Symptoms scale of BR23 was negatively associated with pain (OR=0.11; 95\% CI 0.21 to 0.73$)$. Patients with good functionality-C30 were less likely to experience pain (OR 0.66 $95 \%$ CI (0.15 to 0.75$))$. There was no statistically significant association with axillary web syndrome.

Conclusions In this study pre-treatment QOL in women with breast cancer was a useful predictor of early post-operative complications.

\section{P2-81 THE IMPACT OF SOCIAL ENVIRONMENT ON EARLY COMPLICATIONS IN WOMEN WITH BREAST CANCER IN A REFERENCE CENTRE IN BRAZIL}

doi:10.1136/jech.2011.142976i.16

${ }^{1} \mathrm{D}$ B Ferreira, ${ }^{* 1}$ R Koifman, ${ }^{2}$ A Bergmann. ${ }^{1}$ National School of Public Health, Oswaldo Cruz Foundation, Rio de Janeiro, Brazil; ${ }^{2}$ National Cancer Institute, Rio de Janeiro, Brazil

Introduction Studies suggest that good social support is associated with a reduction in post-treatment complications in breast cancer. The aim of this study is to evaluate the association between pretreatment social support and early complications after surgery (pain and subjective symptoms related to early oedema in the upper limbs) in women with breast cancer.

Methods Participants were women with breast cancer and that presented to the Brazilian National Cancer Institute between June 2009 and March 2010. MOS-SSS was used to assessment of social support before oncology treatment. Information about early postoperative complications were obtained by physical examination 30 days after surgery and collected in a standardised form. Bivariate analysis between dichotomous outcome and selected independent variables were conducted and RRs with $95 \%$ CIs were presented.

Results 181 eligible women were studied. Patients with good emotional and affective support had a $76 \%$ (95\% CI 0.10 to 0.57 ) reduced risk and $62 \%$ (0.12 to 0.64$)$ reduced risk of pain respectively. Better material support was associated with a $73 \%$ (0.11 to 0.623 ) reduction in the risk of pain. No significant association with early oedema was observed.

Conclusions Good social support was associated with reduced risk of post-operative complications in women undergoing treatment for breast cancer.

\section{P2-82 PREGNANCY, EXPOSURE TO PESTICIDES AND INFANT LEUKAEMIA IN BRAZIL}

doi:10.1136/jech.2011.142976i.17

${ }^{1} \mathrm{~J} D$ Ferreira, ${ }^{*} \mathrm{~A}$ C Couto, ${ }^{2} \mathrm{M}$ do Socorro Pombo-de-Oliveira, ${ }^{1} \mathrm{~S}$ Koifman. ${ }^{1}$ National School of Public Health, Oswald Cruz Foundation - Fiocruz, Rio de Janeiro, Brazil; ${ }^{2}$ National Institute of Cancer - Inca, Rio de Janeiro, Brazil

Infant Leukaemia is a rare haematological neoplasm associated with MLL gene rearrangements. Maternal exposure during pregnancy to pesticides, hormones, dipyrone, and topoisomerase-II DNA inhibitors and birth weight were associated risk factors. This is a hospitalbased multicenter case-control study, and were interviewed mothers of 252 IL cases and of 423 controls. Data were obtained regarding environmental maternal exposure during periconceptional, pregnancy and breast-feeding periods, including exposure to pesticides. Unconditional logistic regression was performed and ORs on the association between maternal pesticides exposure and IL, including their $95 \%$ CIs, were ascertained after adjustment to hormonal intake during pregnancy, mother's age, mother's level of education, birth weight and infant's skin colour. An adjusted OR, 2.39, 95\% CI 1.63 to 3.51 was observed for the association between IL and maternal exposure to pesticides during pregnancy, being higher for acute myeloid leukaemia (adjusted OR 3.50, 95\% CI 2.01 to 6.11). The use of pyrethroids during pregnancy revealed an OR 2.18 (95\% CI 1.44 to 3.29), while the use of other pesticides showed an OR $3.61(95 \%$ CI 1.69 to 7.73 ). The reported household use of pesticides revealed an adjusted OR 2.25 (95\% CI 1.48 to 3.43 ), and agriculture exposure showed an adjusted OR 9.26, (95\% CI 2.82 to 30.4). Mixed exposure to different pesticides showed an adjusted OR, 3.83 (95\% CI 1.33 to 11.0) and the exposure to different chemical classes revealed an adjusted OR, 8.87 (95\% CI 1.55 to 50.6). The observed results seem to support the hypothesis that mothers' household use of pesticides and other contaminants may be involved in the aetiology of infant leukaemia.

\section{P2-83 ENVIRONMENTAL EXPOSURES AND CHILDHOOD LEUKAEMIA: AN EXPLORATORY ANALYSIS IN BRAZIL}

doi:10.1136/jech.2011.142976i.18

${ }^{1} \mathrm{~J} D$ Ferreira, ${ }^{*} \mathrm{~A}$ C Couto, ${ }^{2} \mathrm{M}$ do Socorro Pombo-de-Oliveira, ${ }^{1} \mathrm{~S}$ Koifman. ${ }^{1}$ National School of Public Health, Oswald Cruz Foundation - Fiocruz, Rio de Janeiro, Brazil, ${ }^{2}$ National Institute of Cancer - Inca, Rio de Janeiro, Brazil

The aim of this study was to explore the unapparent relationships that several factors related to environmental exposures and individual characteristics existing in our environment seem to be involved with childhood leukaemia. From a database of clinical and epidemiological data obtained from hospital-based multicenter casecontrol study on risk factors for childhood leukaemia, exploratory multivariate analysis was performed the principal component and factor analysis. Further, an unconditional logistic regression was carried out aiming to ascertain the magnitude of association between the selected factors, and their composing variables, with childhood leukaemia. The model displaying the highest power explained $52 \%$ of the total variance, including three factors, each one 